\title{
Dolichol Content and Isoprenolog Distribution in Developing Rat Small Intestine ${ }^{1}$
}

\author{
MARCIA J. ARMSTRONG, ROBERT K. MONTGOMERY, ARTURO PEREZ, AND \\ RICHARD J. GRAND
}

\begin{abstract}
Department of Physiology, University of Massachusetts Medical Center, Worcester [M.J.A.]; and Division of Pediatric Gastroenterology and Nutrition, Floating Hospital for Infants and Children, New England Medical Center, and Department of Pediatrics, Tufts University School of Medicine,
\end{abstract}

Boston, Massachusetts [R.K.M., A.P., R.J.G.]

\begin{abstract}
We examined developmental changes in dolichol concentration and isoprenolog distribution during the postnatal development of rat small intestine. We isolated dolichols from rat small intestinal lipids and quantified the isoprenologs by high performance liquid chromatography. The concentration of intestinal dolichol was highest in the intestine of the newborn rat and lowest in intestine from 19-day-old and lactating female rats. The predominant isoprenolog at all ages examined was that with 18 isoprene units. There was a significant increase in isoprenolog 17 during the 3rd wk of postnatal development and an increase in isoprenolog 19 in the adult rat small intestine. The timing of the changes in dolichol content suggests that this might be an important control point in intestinal glycoprotein synthesis during development. (Pediatr Res 21: 261265,1987 )
\end{abstract}

\section{Abbreviations}

HPLC, high performance liquid chromatography TLC, thin-layer chromatography

ANOVA, two-way analysis of variance

During a brief period in the 3rd week of postnatal life, the morphology and biochemistry of the rat enterocyte undergo a profound reorganization (1). Several digestive enzymes such as sucrase-isomaltase and alkaline phosphatase, which are glycoproteins situated at the luminal cell surface, increase dramatically in activity at this time. Although regulatory factors such as glucocorticoids and thyroxine are known to influence the expression of these enzymes, the molecular events that occur still remain largely unexplained. A common feature shared by these enzymes is that approximately $15-20 \%$ of the enzyme molecule is carbohydrate (2).

There is increasing evidence that a significant portion of the carbohydrate of sucrase-isomaltase, as well as other intestinal cell surface glycoproteins, consists of asparagine-linked, complex oligosaccharides. The carrier lipid dolichol is known to play a key role as an intermediate in synthesis of glycoproteins of this type (3). Drugs that interfere with the dolichol-mediated step in glycoprotein synthesis have been demonstrated to block devel-

Received August 14, 1986; accepted October 23, 1986.

Correspondence to Dr. Richard J. Grand, Pediatric Gastroenterology/Nutrition, Box 213, New England Medical Center, 750 Washington Street, Boston, MA 02111 .

Supported in part by NIH Research Grants AM 06514 (M.J.A.), AM 31790 (M.J.A.), HD 14498 (R.K.M.), AM 14523 (R.J.G.), and AM 32658 (R.J.G.).

Presented in part at the American Gastroenterological Association Meeting, Chicago, May 1982. opment $(4,5)$. In addition, dolichol and dolichyl phosphate synthesis have been shown to change during the course of development (6-8).

The dolichols are a family of isoprenoid alcohols consisting of multiple unsaturated isoprene units with an alpha-terminal alcohol and a saturated $\alpha$-terminal isoprene unit. Dolichols serve as the carrier of the core carbohydrate, dolichol-P-P-GlcNAcGlcNAc-mannose, in glycoprotein synthesis as well as of the intermediate dolichol-P-mannose necessary for the addition of mannose to the core carbohydrate (3). Although mammalian tissues have been shown to contain dolichols of 16-23 isoprene units, the size of the specific dolichol which serves the carrier function is unknown (9). Furthermore, little is known about developmental changes in dolichol content or distribution patterns of dolichol isoprenologs in the small intestine.

We have modified existing methodology for isolation of dolichols from rat small intestine and the determination of the distribution of dolichol isoprenologs. We have utilized this methodology to examine dolichol isoprenolog patterns in developing rat small intestine and to quantify dolichol content at different ages during postnatal development.

\section{METHODS}

Chemicals. Authentic pig liver dolichol, dolichyl acetate, and dolichyl phosphate were obtained from Sigma (St. Louis, MO). Polyprenols were obtained from Calbiochem-Behring (La Jolla, CA). HPLC grade solvents (isopropanol, methanol) were obtained from Waters Associates (Milford, MA). All other reagents were from Fisher Scientific (Pittsburgh, PA).

Animals. CD strain Sprague-Dawley rats were obtained from Charles River Breeding Laboratories, Wilmington, MA. The animals were housed in rooms with a constant light/dark cycle and maintained on standard laboratory rat Chow (Ralston Purina, St. Louis, MO) and water ad libitum. To determine whether the quantity of dolichols or the distribution of dolichol isoprenologs changed during intestinal development, we examined dolichols from the intestines of newborn, 14-day-old, 19-day-old, and adult male rats. Since the intestine of lactating females undergoes significant hyperplasia, dolichols from that source were also characterized.

Isolation of dolichols. Preparations of the rat small intestine which included the intestinal mucosa, submucosa, and muscularis ("whole intestine") were made from newborn and adult rats. Preparations of the intestinal mucosa alone were isolated from individual 14-day old, 19-day old, adult male and lactating female rat intestine. The mucosa scraped from the entire length of the small intestine was homogenized in $0.9 \%$ saline, mixed well, and separated into aliquots containing $0.6 \mathrm{~g}$ mucosal tissue for subsequent lipid extraction. Tissue lipids were extracted according to the method of Folch et al. (10). The total lipid 
content of the mucosal and intestinal homogenates ranged from 0.7 to $1.2 \mathrm{mg} / \mathrm{g}$ tissue. The extracted tissue lipids were saponified in freshly prepared saturated ethanolic-potassium hydroxide for $3 \mathrm{~h}$ at $60^{\circ} \mathrm{C}$ followed by extraction three times with petroleum ether $(11) .\left[{ }^{3} \mathrm{H}\right]$-cholesterol $(100,000 \mathrm{cpm})$ was added to an aliquot of homogenate as a marker for the methodological loss of nonsaponifiable lipid during the purification procedure. Cholesterol recoveries were $80.6 \pm 12.0 \%$. Although cholesterol and dolichol are structurally unique compounds, both are nonpolar hydrocarbons with very limited aqueous solubilities. Consequently, cholesterol and dolichol would be expected to partition between aqueous and organic phases in a similar fashion. ${ }^{3} \mathrm{H}-$ cholesterol should thus provide a reasonable approximation of the recovery of nonsaponifiable lipids due to losses of solvent during the procedure.

Sample purification: Sep-Pak. Nonsaponifiable lipids were eluted from Sep-Pak ODS cartridges (Waters Associates) with sequential applications of $100 \%$ methanol $(5 \mathrm{ml}) ; 60 \%$ methanol-40\% isopropanol (2 $\mathrm{ml}) ; 40 \%$ methanol-60\% isopropanol $(5 \mathrm{ml})$. The eluates were dried under $\mathrm{N}_{2}$ and resuspended in isopropanol prior to chromatographic characterization. These Sep-Pak cartridges are essentially small chromatography columns filled with C18-coated silica gel. Since the Sep-Paks are chemically similar to the HPLC column they can be utilized to perform initial precolumn separation of compounds from complex mixtures by differential solvent elution. TLC of these eluates demonstrated that cholesterol and trace quantities of a substance that cochromatographed with dolichyl phosphate standard eluted from the Sep-Pak with $100 \%$ methanol (first eluent) while the dolichols eluted with $40 \%$ methanol-60\% isopropanol (third eluent). No dolichols eluted from the Sep-Pak with either the first eluent or the second eluent (60\% MeOH:40\% isopropanol). A third step reduced the amount of other nonsaponifiable lipids to levels which permitted baseline resolution of the dolichols.

HPLC of dolichols: analytical column. The individual dolichol isoprenologs were resolved by reversed-phase HPLC using a Beckman Altex gradient liquid chromatograph (model 330, Beckman Instruments, Palo Alto, CA) equipped with two model $110 \mathrm{~A}$ pumps and a model 210 injector. The eluate from an Ultrasphere ODS (C18) column (Altex Instruments, Palo Alto, $\mathrm{CA}, 4.6 \times 250 \mathrm{~mm}$ ) was monitored with a Hitachi Model 10040 (Hitachi Instruments, Ltd., Tokyo, Japan) UV-vis variable wavelength detector set at $211 \mathrm{~nm}$. The elution profile was recorded and peak areas integrated with a Shimadzu CR1-A (Shimadzu Corp., Kyoto, Japan) integrating recorder. Prior to the injection of the sample, the reversed-phase column was equilibrated with $70 \%$ isopropanol:30\% methanol at a flow rate of $0.7 \mathrm{ml} / \mathrm{min}$. The mobile phase was changed via a step-gradient to $100 \%$ isopropanol for sample elution from the column.

HPLC of dolichols: preparative column. The isolation of larger quantities of individual dolichol isoprenologs for mass spectroscopy was performed on a preparative Ultrasphere ODS column (Beckman Altex, Palo Alto, CA, $10.0 \times 250 \mathrm{~mm}$ ). Dolichols were eluted from the column with $100 \%$ isopropanol at a flow rate of $2.0 \mathrm{ml} / \mathrm{min}$.

$T L C$. TLC was performed on fractions collected from the HPLC column according to Carroll et al. (12) to assess by alternate methodology the composition of the fractions. The HPLC fractions were chromatographed with authentic cholesterol, dolichol, dolichyl phosphate, and dolichyl acetate standards and the developed chromatograms visualized in the presence of iodine vapor.

Mass spectroscopy. The identity of the major peak in pig liver is reported to be dolichol-19 (11). To confirm this in our system, the pig liver standard was chromatographed by preparative HPLC and the fractions containing the major dolichol peak were collected from sequential injections. The isolated peak and a sample of polyprenol-19 were analyzed by mass spectroscopy. These samples were both found to have a mass ion of 1311 , consistent with an empirical formula of $\mathrm{C}_{95} \mathrm{H}_{154} \mathrm{O}$ or 19 isoprene units, thus confirming the identity of the major standard peak.

Quantification of integrated peak area. The peaks of the elution profile of the dolichol isoprenologs were integrated using the computing integrator. The resulting integrated peak area was proportional to the dolichol concentration contained in the column effluent as it passed the UV detector. Since it could not be assumed that two compounds with differing numbers of carbon-carbon double bonds would exhibit equal absorbances at $211 \mathrm{~nm}$ at equal concentrations, we prepared standard solutions of polyprenols with 16,17 , and 19 isoprene units $(\mathrm{C} 80, \mathrm{C} 85$, and C95) and plotted integrated peak areas obtained at $211 \mathrm{~nm}$ versus polyprenol concentration injected. The resulting standard curves were superimposable (Fig. 1). Since both polyprenol-19 and dolichol-19 had identical mass ions of 1311 , and cochromatographed under our HPLC conditions, we assumed that the standard curves obtained from the chromatography of the polyprenol isoprenologs could justifiably be used to estimate the quantity of dolichol isoprenologs with 16-20 isoprene units in our purified intestinal lipid samples, with a detection limit of 50 ng.

Dolichol recovery experiments. In order to assess the overall loss of dolichol as well as the loss of individual dolichol isoprenologs during lipid extraction, saponification, and Sep-Pak analysis, recovery experiments were performed. A gravimetrically determined amount of pig liver dolichol standard was carried through the purification steps outlined above. Following elution of the purified dolichols from the Sep-Pak, HPLC analysis was performed. The peaks were quantified as outlined above and compared to values obtained when pig liver dolichols were subjected to HPLC without intermediate processing. The percent distribution of the dolichol isoprenologs contained in both samples was identical, indicating that there was no preferential loss of one or more of the isoprenologs.

A second recovery experiment was performed in which a known quantity of dolichol standard was added to a quantity of

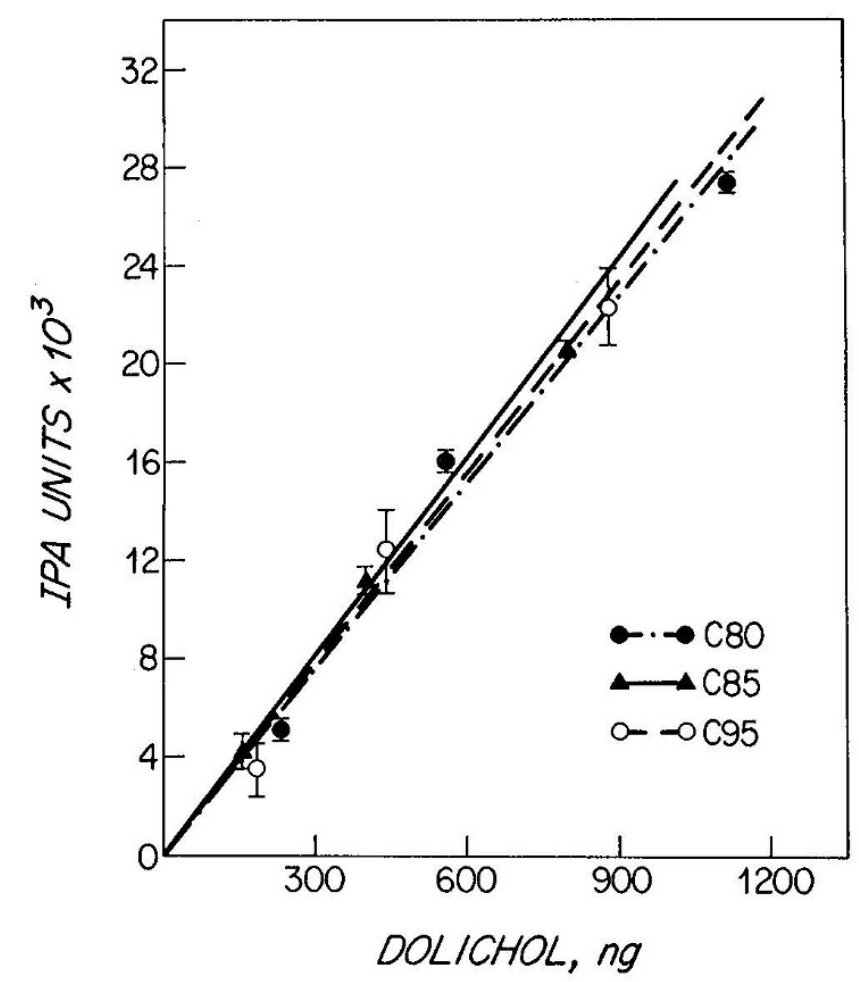

Fig. 1. Standard curves for integrated peak area $(I P A)$ versus dolichol concentration. Curves were constructed by plotting integrating peak areas versus nanograms of dolichol injected for $\mathrm{C} 80, \mathrm{C} 85$, and $\mathrm{C} 95$ dolichols. 
lipid extract identical to those used in the remainder of the study. Purification was performed in parallel with an unspiked sample of lipid extract. The lipid mixtures were saponified, eluted from a Sep-Pak cartridge, and chromatographed by HPLC. The quantity of dolichol obtained by HPLC analysis accounted for $97 \%$ of the added dolichol standard plus the dolichol contained in the tissue. Thus, the procedures used to isolate dolichols from the intestinal mucosa did not result in the preferential loss of either individual dolichol isoprenologs or total dolichol mass when combined with nonsaponifiable lipids.

Statistical analysis. Group means were analyzed by ANOVA. The ANOVA for both dolichol concentration and percent distribution was significant for both age and dolichol isomer. Individual group means were examined for specific contribution to parametric multiple range test (13).

\section{RESULTS}

Distribution of intestinal dolichol isoprenologs on HPLC: $m u$ cosa. HPLC retention times of the dolichols obtained from the purified adult rat small intestine were compared to the retention

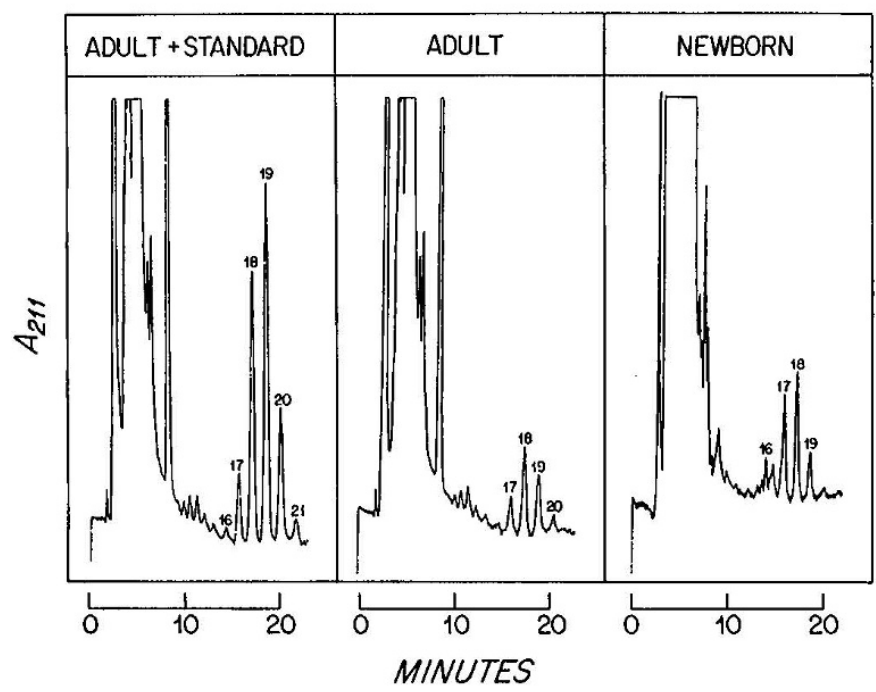

Fig. 2. HPLC of rat intestinal dolichols (see "Methods" for details). The left panel demonstrates cochromatography of the pig liver standard dolichols with dolichols obtained from adult rat intestine. The center and right panels show chromatograms of adult and newborn dolichols, respectively. The numbers refer to the number of isoprene units in the dolichol isoprenolog represented by each peak. times of authentic pig liver dolichol standards. Intestinal dolichols cochromatographed with the major standard peaks; the retention times of the unknown peaks were identical to those of the standard peaks, with additive peak heights and no shoulders on the peaks (Fig. 2).

The purified dolichols obtained after Sep-Pak elution were analyzed by HPLC. From identification of the major liver standard peak and comparison of retention times, the adult intestinal mucosa was found to contain dolichol isoprenologs with 17,18 , 19 , and 20 isoprene units. The major dolichol isoprenolog was that with 18 isoprene units which comprised $47.1 \pm 3.0 \%$ of the total integrated peak area (Fig. 2).

Dolichol content of tissue. The total concentration of dolichol, expressed as $\mu \mathrm{g}$ dolichol/g tissue, ranged from $7.6 \pm 3.1$ in the lactating females to $55.7 \pm 28.4$ in the newborn intestine (Table 1). These quantities compare favorably with those reported recently by Eggens et al. (14) for rat intestine $(16.6 \pm 1.7 \mu \mathrm{g} / \mathrm{g})$ using HPLC. Adair and Keller (9) reported values of $19 \mu \mathrm{g} / \mathrm{g}$ tissue using a different method of analysis and Rip et al. (15) obtained values ranging from $23.2-165 \mu \mathrm{g} / \mathrm{g}$ for six rat tissues using HPLC.

Because of the small size of the newborn intestine, the mucosa was analyzed along with the underlying muscularis. To test whether the inclusion of all intestinal layers altered the dolichol concentration in the intestine, we assayed the dolichol concentration in whole adult intestine as well as in the intestinal mucosa. Dolichol concentrations were not significantly different $(25.6 \pm$ 9.9 versus $30.9 \pm 8.5 \mu \mathrm{g} / \mathrm{g}$ tissue, mucosa versus whole intestine). The pattern of dolichol molecular species distribution in the adult whole intestine was more similar to that of the adult mucosa than to the newborn whole intestine (Fig. 3). Thus, in the newborn, the appearance of detectable amounts of the dolichol16 and the markedly increased concentration of dolichols do not appear to be an artifact of the tissue preparation used in the assay.

Dolichols in development. Dolichols were isolated from the intestinal mucosa of 14-day-old suckling, 19-day-old weanling, lactating female rats, and from the whole intestine of newborn rats (Fig. 3). In all cases, the major molecular species of dolichol (between 42 and 53\%) was dolichol-18. All animals studied also had dolichols of 17 (18-35\%) and 19 (15-20\%) isoprene units. However, both the adult males and the 14-day-old suckling rats had detectable quantities of dolichol-20. The pattern of dolichols from the whole intestine of newborn rats was found to have a detectable amount of dolichol-16 (8.6 $\pm 5.2 \%)$ although the major dolichol species was again dolichol-18 $(50.7 \pm 5.8 \%)$. The small peaks of dolichol-20 in the newborn and dolichol-16 in the adult were not separated as peaks from the background by the HPLC integrator. Known amounts of pig liver dolichol standard

Table 1. Concentration of molecular species of rat intestinal dolichol homologs*

\begin{tabular}{|c|c|c|c|c|c|c|c|}
\hline & \multicolumn{7}{|c|}{ No. of isoprene units $(\mu \mathrm{g} / \mathrm{g}$ tissue $\pm \mathrm{SD})$} \\
\hline & $\mathrm{N}$ & 16 & 17 & 18 & 19 & 20 & TOTAL \\
\hline \multirow[t]{2}{*}{ Newborn } & 8 & 5.1 & $16.3^{1}$ & $28.3^{2}$ & $6.7^{3}$ & ND & $55.7^{4}$ \\
\hline & & \pm 6.0 & \pm 9.5 & \pm 13.8 & \pm 2.8 & & \pm 28.4 \\
\hline \multirow[t]{2}{*}{ 14-day-old } & 6 & $\mathrm{ND} \dagger$ & 6.3 & 13.2 & 4.8 & 1.9 & 25.3 \\
\hline & & & \pm 2.2 & \pm 3.1 & \pm 2.6 & \pm 1.4 & \pm 7.1 \\
\hline \multirow[t]{2}{*}{ 19-day-old } & 4 & ND & 3.7 & 5.4 & 1.4 & ND & 10.6 \\
\hline & & & \pm 2.9 & \pm 5.5 & \pm 0.4 & & \pm 8.5 \\
\hline \multirow[t]{2}{*}{ Adult male mucosa } & 5 & ND & 5.8 & 12.1 & 7.3 & 2.6 & 25.6 \\
\hline & & & \pm 3.1 & \pm 4.6 & \pm 2.8 & & \pm 9.9 \\
\hline Adult male whole intestine & 2 & ND & 9.4 & 14.1 & 6.4 & 1.1 & 30.9 \\
\hline \multirow[t]{2}{*}{ Adult female lactating } & 5 & ND & 1.6 & 3.9 & 2.1 & ND & 7.6 \\
\hline & & & \pm 0.9 & \pm 1.6 & \pm 0.7 & & \pm 3.1 \\
\hline
\end{tabular}

* Column comparisons: 1. lactating vs NB, $p<0.05 ; 2.19$ day $v s \mathrm{NB}, p<0.05$, lactating $v s$ NB, 14 day, $p<0.05 ; 3.19$ day $v s$ NB, adult $p<$ 0.05 , lactating vs NB $p<0.05 ; 4.19$ day $v s \mathrm{NB}, p<0.05$, lactating $v s \mathrm{NB}, 14$ day, $p<0.05$. All other comparisons not significant.

$\dagger$ Not detectable. 


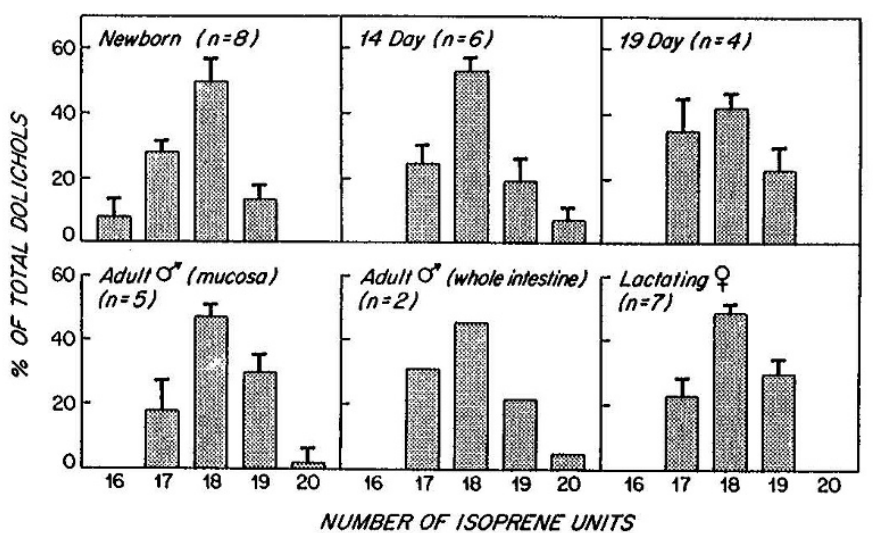

Fig. 3. Distribution patterns of the molecular species of intestinal dolichol isoprenologs in newborn, 14-day-old, 19-day-old, adult male and lactating female rats. Data are mean \pm SEM.

containing both dolichol-16 and dolichol-20 were added to the adult and newborn samples. The combination of adult unknown with known amounts of standard did not increase the peak of dolichol-16 nor did newborn unknown increase the dolichol-20 peak. This finding suggested to us that the newborn intestine contains dolichol-16, but not dolichol-20, and that the adult has dolichol-20 but not dolichol-16.

ANOVA demonstrated that the high concentration of dolichol measured in the newborn intestine was significantly greater than that in the 19-day-old rats. The concentrations of dolichol in the 14-day, 19-day, and adult males were not significantly different from each other (but the lactating females had significantly lower tissue concentration of dolichol than the newborn and 14-dayold groups). The 19-day-old rat small intestine had a significantly greater percentage of dolichol-17 and -19 , and significantly less dolichol-18 than did the newborn rat small intestine. During development, the percent of dolichol-17 peaked in the 19-dayold rat small intestine, while the percentage of dolichol-19 peaked in the adult small intestine (Fig. 4).

\section{DISCUSSION}

We have utilized a rapid method for the isolation and identification of dolichol homologs from individual rat intestines. In addition to identifying the isoprenologs, we have quantified each in rat intestine during development. Our data demonstrate that at all ages examined, the predominant isoprenolog has 18 isoprene units, with the 17 and 19 isoprene unit isoprenologs making up most of the remainder. A 16 isoprene unit dolichol was identified only in newborn intestine, but was not detected at any other age examined. The lower limit of detection was $50 \mathrm{ng}$, making it unlikely that the absence of this isoprenolog in other age groups represents a failure to detect significant quantities. Intestine from the newborn animals had significantly higher concentrations of dolichol than the others, while the lactating females and 19-day (suckling) animals had the lowest.

The HPLC chromatograms of the pig liver dolichol isoprenologs are similar to those obtained by other authors using different columns and mobile phases. Pig liver has been reported to have dolichols of 17 to 20 isoprene units, with dolichol-19 preponderant. Human liver has dolichols of 17 to 23 isoprene units, with dolichol-19 and dolichol-20 most abundant (11). Human tissues generally have higher levels of dolichol than the corresponding rat tissue (16). Rip et al. (15) examined the distribution of dolichol isoprenologs in six rat tissues and found tissue-specific patterns; dolichol-18 and dolichol-19 were predominant, although their relative abundance varied from tissue to tissue.

It is unknown whether the dolichyl phosphate intermediate in protein glycosylation is a dolichol isoprenolog of a particular

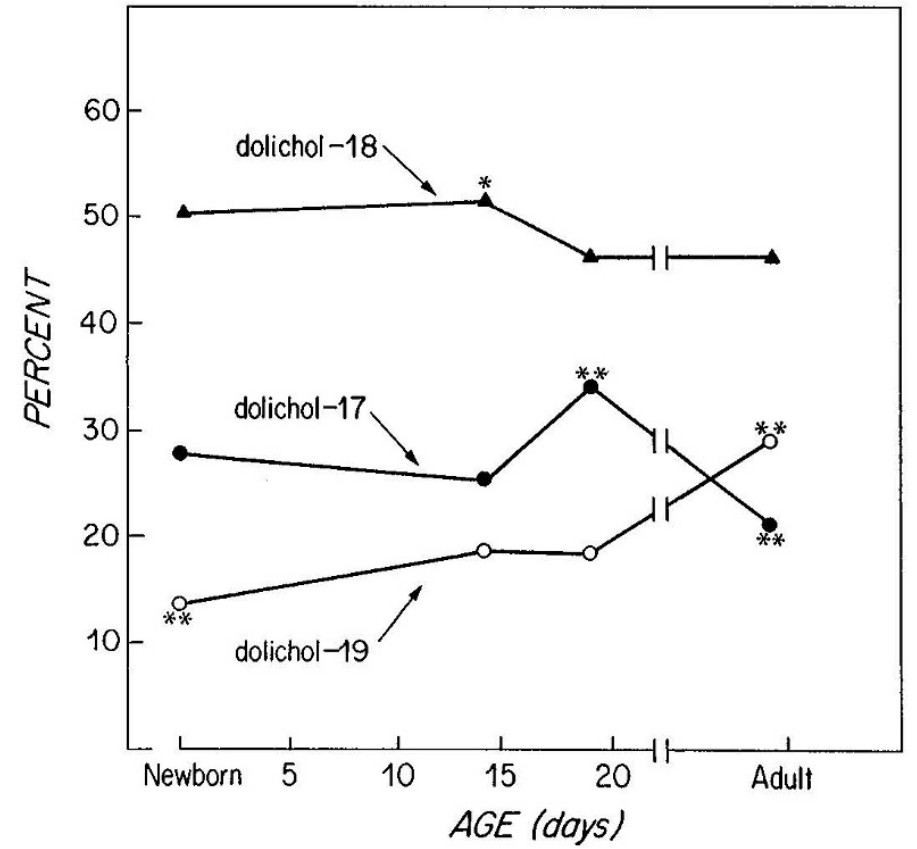

Fig. 4. Developmental changes in dolichol isoprenologs. Mean distribution of dolichol isoprenologs is plotted against age of animals. For each isoprenolog, the $p$ values refer to comparisons between animals of different ages. ${ }^{*} p<0.05$ versus 19 day and adult; ${ }^{* *} p<0.05$ versus all

length, or if dolichol length is unimportant. However, the distribution of dolichol isoprenologs in human hepatocarcinoma has been found to be altered (14). Since abnormal glycoproteins are associated with this pathological state, there may be a relationship between dolichol isoprenolog pattern and protein glycosylation. We therefore examined isoprenolog patterns during intestinal development. Our data demonstrate that intestinal total dolichol content decreases during the $3 \mathrm{rd}$ wk of postnatal development. This decrease is largely accounted for by decreases in dolichol18 and dolichol-19. Simultaneously, there was a significantly increased percentage of dolichol-17 in the 19-day-old rat intestine. Since it occurs at the same time that major changes in disaccharidase levels are taking place (1), this change in distribution of dolichols may represent an important regulatory point.

The dolichols we have examined are the free alcohols obtained by saponification of total tissue lipids. Although the active intermediate in glycoprotein synthesis is dolichyl phosphate (3), the free dolichols constitute the largest tissue pool, and we have concentrated our efforts upon them. Rossignol et al. (8) demonstrated that in the development of sea urchins, the free alcohol is the major end-product of de novo synthesis, and that this pool of preformed dolichol in turn serves as the source of dolichol for phosphorylation. In their experiments, synthesis of dolichyl phosphate continued for $6 \mathrm{~h}$ after the de novo synthesis of dolichol was blocked. However, Ekstrom et al. (17) have suggested that, in the rat liver, free dolichol and dolichyl phosphate are not synthesized by a common pathway and as such no precursorproduct relationship exists between the two compounds. Our data suggest that there is a decrease in the tissue concentration of dolichol at the time of a major burst of dolichyl phosphatedependent glycosylation. One interpretation of these data is that the free dolichols are being converted to dolichyl phosphate, a finding in agreement with those of Rossignol et al. (8).

Inhibition of protein glycosylation by tunicamycin leads to developmental abnormalities at specific stages during sea urchin embryogenesis (18). Since this drug specifically inhibits dolichol- 
mediated glycosylation, these data suggest a specific requirement for glycoprotein synthesis during sea urchin gastrulation. Further, compactin, a drug that blocks polyisoprenoid synthesis, had the same effect, producing abnormal gastrulation of sea urchins without affecting earlier development or concurrent synthesis of DNA, RNA, or proteins. The effect could be prevented by the simultaneous addition of dolichol, thus demonstrating the key role of dolichol in development (4).

At birth, the small intestine is adapted to a diet of milk and during the rapid growth of the suckling period, the small intestine maintains a high level of lactase (19). During the weaning period, the intestinal epithelium undergoes a profound reorganization during which new glycoproteins appear and old ones decline in abundance, as the intestine adapts to an adult diet (1). While our observed changes in dolichol concentration and percent distribution do not in themselves demonstrate a functional role for dolichol in intestinal differentiation, they do occur at significant developmental stages and suggest that synthesis of dolichol and dolichyl phosphate may be a regulatory point in intestinal development at one or more stages. With the methodology developed herein, direct experiments to test this question are now possible.

Acknowledgment. The mass spectoscopy was generously performed by Dr. Catherine Costello at the Massachusetts Institute of Technology National Mass Spectrometry Facility which is supported by the NIH Division of Research Resources (Grant RR03171 to K. Biemann, Ph.D.).

\section{REFERENCES}

1. Moog F 1979 The differentiation and redifferentiation of the intestinal epithelium and its brush border membrane. CIBA Symposium 70:31-50

2. Montgomery RK, Jonas MM, Grand RJ 1982 Intestinal disaccharidases: structure, function and deficiency. In: F Lifshitz, (ed.), Carbohydrate Intolerance in Infancy, Marcel Dekker, Inc., New York, pp 75-92
3. Hubbard SC, Ivat RJ 1981 Synthesis and processing of asparagine-linked oligosaccharides. Ann Rev Biochem 50:555-593

4. Carson DD, Lennarz WJ 1979 Inhibition of polyisoprenoid and glycoprotein biosynthesis causes abnormal embryonic development. Proc Natl Acad Sci USA 76:5709-5713

5. Sarras MP, Maylie-Pfenninger M, Manzi RM, Jamieson JD 1981 The effect of tunicamycin on development of the mammalian embryonic pancreas. Devel Biol 87:1-15

6. James MJ, Kandutsch AA 1980 Elevated dolichol synthesis in mouse testes during spermatogenesis. J Biol Chem 255:16-19

7. Lucas JJ, Levin E 1977 Increase in the lipid intermediate pathway of protein glycosylation during hen oviduct differentiation. $J$ Bilol Chem 252:43304336

8. Rossignol DP, Scher M, Waechter CJ, Lennarz WJ 1983 Metabolic interconversion of dolichol and dolichyl phosphate during development of the sea urchin embryo. J Biol Chem 258:9122-9127

9. Adair WL, Keller RK 1982 Dolichol metabolism in rat liver. J Biol Chem 257:8990-8996

10. Folch J, Lees M, Stanley GHS 1957 A simple method for the isolation and purification of total lipids from animal tissues. J Biol Chem 226:497-510

11. Freeman DJ, Rupar CA, Carroll KK 1980 Analysis of dolichol in human tissues by high pressure liquid chromatography. Lipids 15:191-193

12. Carroll KK, Vilim A, Woods MC 1973 Polyprenols of beef and human pituitary glands. Lipids 8:246-248

13. Schefler WC 1979 Statistics for the Biological Sciences, 2nd ed. AddisonWesley Publishing Company, inc., Reading, MA, pp 136-140

14. Eggens I, Chojnacki T, Keene L, Dallner G 1983 Separation, quantitation and distribution of dolichol and dolichyl phosphate in rat and human tissues. Biochim Biophys Acta 751:355-368

15. Rip JW, Chaudhary N, Carroll KK 1983 Distribution and metabolism of dolichol and dolichyl phosphate in rat liver. Canad J Biochem 61:10251031

16. Rupar CA, Carroll KK 1978 Occurrence of dolichol in human tissues. Lipids 13:291-293

17. Ekstrom TJ, Chojnacki T, Dallner G 1984 Metabolic labeling of dolichol and dolichyl phosphate in isolated hepatocytes. J Biol Chem 259:10460-10468

18. Schneider EG, Nguyen HT, Lennarz, WJ 1978 The effect of tunicamycin, an inhibitor of protein glycosylation, on embryonic development in the sea urchin. J Biol Chem 253:2348-2355

19. Doell RG, Kretchmer N 1962 Studies of small intestine during development. I. Distribution and activity of beta-galactosidase. Biochim Biophys Acta 62:353-362 